\title{
A TEORIA TRANSCULTURAL E SUA APLICAÇÃO EM ALGUMAS PESQUISAS DE ENFERMAGEM: UMA REFLEXÃO
}

Transcultural theory and its application in some

research of nursing: a reflection

Teoría Transcultural y su uso en algunas

investigaciones de enfermería: una reflexión

Maria Aparecida Vasconcelos Moura

Rosilda Alves da Silva Isla Chamilco

Leila Rangel da Silva

\section{Resumo}

Esta é uma reflexão teórica, elaborada a partir de referencial bibliográfico específico e experiências das autoras na prática da enfermagem, sobre os fundamentos da Teoria da Diversidade e Universalidade do Cuidado Cultural. Trata da tentativa de estabelecer uma vinculação entre a aplicação dos conhecimentos adquiridos no campo da Enfermagem e esta abordagem teórica. Discute-se as relações entre a prática da enfermagem, os ritos e os fundamentos desta Teoria e sua aplicação nas pesquisas de enfermagem. 0 levantamento bibliográfico foi realizado por meio de busca eletrônica e manual em acervos de bibliotecas. As análises foram pautadas no detalhamento dos fundamentos teóricos desta abordagem e pesquisas fundamentadas nesta Teoria. A conclusão destaca que os fundamentos desta Teoria podem descrever a totalidade dos fatores, diferentes mas próximos e integrados, que compõem a estrutura sóciocultural do cuidado de enfermagem e a visão de mundo das pessoas que o recebem.

Palavras-chave: Enfermagem Transcultural. Teoria de Enfermagem. Modelos de Enfermagem.

$$
\text { Pesquisa em Enfermagem. }
$$

\begin{abstract}
This is a theoretical reflection, elaborated from specific bibliographical referencial and experiences of the authors in the practical one of the nursing, on the beddings of the Theory of the Diversity and Universality of the Cultural Care. It deals with the attempt to establish an entailing enters the application of the acquired knowledge in the field of the Nursing and this theoretical boarding. One argues the relations between the practical one of the nursing, the rites and the beddings of this Theory and its application in the nursing research. The bibliographical survey was carried through by means of electronic and manual search in quantities of libraries. The analyses had been pautadas in the detailing of the theoretical beddings of this boarding and research based on this Theory. The conclusion detaches that the beddings of this Theory can describe the totality of the factors, different but next and integrated, that compose the sóciocultural structure of the care of nursing and the vision of world of the people who receive it.
\end{abstract}

\section{Resumen}

Esta es una refleción teórica, elaborada a partir de referencial bibliográfico específico y de experiencias de las autoras en la práctica de la enfermería, sobre los fundamentos de la Teoría de la Diversidad y Universalidad del Cuidado Cultural. Se trata de la tentativa de establecer una vinculación entre la aplicación de los conocimientos adquiridos en el área de la enfermería y este enfoque teórico. Se discute las relaciones entre la práctica de la enfermería, los ritos y los fundamentos de esta Teoría y su aplicación en las pesquisas de enfermería. El inventario bibliográfico fue cumplido por medio de busca electrónica y manual en acervos de bibliotecas. Los análisis fueron pautados en la pormenorización de los fundamentos teóricos de este enfoque y encuestas basadas en esta Teoría. La conclusión destaca que los fundamentos de esta Teoría pueden describir la totalidad de los factores, diferentes pero próximos e integrados, que componen la estructura sociocultural del cuidado de enfermería y la visión de mundo de las personas que lo reciben.

Palabras clave:

Transcultural Nursing. Nursing Theory. Models of Nursing. Nursing Research.
Enfermería Transcultural. Teoria de Enfermería. Modelos de Enfermería. Investigación en Enfermería. 


\section{CONSIDERAÇÕES INICIAIS}

Este estudo é uma reflexão teórica, a partir de referencial bibliográfico específico e das experiências das autoras na prática da enfermagem sobre os fundamentos teóricos da Enfermagem Transcultural de Leininger. Seus objetivos são: (a) apresentar os fundamentos da Teoria; (b) destacar aspectos de algumas pesquisas de enfermagem que adotam este referencial.

Para cuidarmos da saúde do ser humano, não devemos somente nos restringir aos conceitos de prevenção, diagnóstico, tratamento e reabilitação. É importante conhecermos o contexto cultural, os valores, as crenças, os rituais e o modo de vida do indivíduo e de suas famílias, numa perspectiva de construção de um novo paradigma para abordagem da saúde e da doença.

Desde a década de 50 , Leininger ${ }^{1}$ uniu os seus conhecimentos adquiridos no campo da enfermagem com os preceitos da antropologia, defendendo a Teoria da Diversidade e a Universalidade do Cuidado Cultural. Para esta autora, a Enfermagem Transcultural é a área da Enfermagem que focaliza o estudo comparativo e a análise de variadas culturas, no que diz respeito ao comportamento relativo ao cuidado geral, ao cuidado de enfermagem.

Vários estudiosos, como Minayo², Langdon ${ }^{3}$, Queiroz ${ }^{4}$, assim como a própria Leininger ${ }^{1}$, destacam e enfatizam a importância de associar a antropologia cultural à área da saúde, por considerarem essa ligação fundamental para o entendimento do processo saúde-doença.

No sentido de formular a Teoria da Diversidade e Universalidade do Cuidado Cultural, Leininger ${ }^{5}$ selecionou e utilizou constructos da antropologia e de cuidado próprio da Enfermagem. Ambos estão contidos em si e formam um todo irredutível, o que representa uma orientação humanística da vida e do viver. Assim sendo, o cuidado é culturalmente constituído. Cada cultura tem a sua forma, seus padrões, suas expressões e estruturas para conhecer, explicar e predizer o estado de bem-estar, assim como padrões de comportamento relacionados ao processo saúde-doença e os universos sociais e culturais onde ocorrem (Leininger ${ }^{5,6}$ ).

Essa Teoria utilizou-se de um conjunto interrelacionado de conceitos e hipóteses de enfermagem fundamentados nas necessidades culturais de indivíduos e grupos, incluindo manifestações de comportamentos relativos ao cuidado, às crenças e aos valores, com a finalidade de realizar um cuidado de enfermagem efetivo e satisfatório. Nessa perspectiva, se a prática da Enfermagem não for considerada em seus aspectos culturais da necessidade humana, conseqüentemente ela exibirá sinais de ineficiência com conseqüências desfavoráveis para aqueles a quem assiste.
A Enfermagem Transcultural tem como foco, o estudo e a análise comparativa de diferentes culturas, no que diz respeito ao comportamento relativo ao cuidado, especialmente ao cuidado de enfermagem. Ela tem o propósito de desenvolver um corpo de conhecimento científico e humanizado capaz de viabilizar a prática do cuidado universal e que seja culturalmente específico. Dessa forma, essa Teoria tem por finalidade tornar o conhecimento e a prática profissional culturalmente embasados, conceituados, planejados e operacionalizados.

A Teoria de Enfermagem Transcultural refere-se a um conjunto de inter-relações de conceitos e hipóteses que respeitam os comportamentos, os valores, as crenças dos indivíduos e dos grupos na execução do cuidado. $\mathrm{E}$ desse modo, reconhecer os aspectos culturais das necessidades humanas, considerando as peculiaridades oriundas do modo de vida de cada indivíduo. Ainda apresenta pressupostos que dão apoio à sua previsão de que culturas diferentes percebem e praticam cuidado de diferentes maneiras, mesmo que alguns elementos comuns existam, em relação ao cuidado em todas as culturas do mundo. Assim, a cultura é definida como valores, crenças, normas e práticas de vida de um determinado grupo, aprendidos, partilhados e transmitidos que orientam o pensamento e as decisões e as ações, de maneira padronizada (Leininger ${ }^{5}$ ).

\section{A PRÁTICA DE ENFERMAGEM, A CULTURA E OS RITOS NA TEORIA TRANSCULTURAL}

A Enfermagem Transcultural objetiva prover uma forma de cuidar que seja sensível e tenha origem nas necessidades do indivíduo, sua família e grupos culturais. Estes devem ser estudados em profundidade para possibilitar um tipo de cuidado culturalmente congruente com as necessidades da população $\left(\right.$ Herberg $\left.^{8}\right)$. Os conhecimentos são construídos com base em estudos comparativos, para identificar similaridades e diferenças sobre 0 cuidar e os comportamentos relativos à saúde (Leininger $\left.{ }^{1}\right)$.

A disposição definida pela Enfermagem Transcultural foi a de proporcionar um serviço de atendimento significativo e eficaz para as pessoas, de acordo com seus valores culturais e seu contexto de saúde-doença. Assim sendo, a Teoria foi construída a partir da premissa de que as pessoas de cada cultura, não apenas podem saber e definir as formas nas quais experimentam e percebem seu mundo de atendimento de enfermagem, mas também podem relacionar essas experiências, percepções e visão de mundo com as suas crenças e práticas de saúde (Leininger ${ }^{1}$ ).

0 pressuposto da Teoria sobre o cuidado como a essência e foco central dominante e unificador da Enferma- 
gem apresenta quatro justificativas, quais sejam: (1) o constructo do cuidado é essencial para o crescimento, o desenvolvimento e a sobrevivência humana; (2) a finalidade é explicar e compreender o papel daquele que cuida e daquele que recebe o cuidado; (3) a preservação e manutenção do cuidado como atributo humano é essencial para a sobrevivência de seres humanos e das culturas através dos tempos; e (4) a Enfermagem é uma profissão que nunca estudou sistematicamente 0 cuidado e sua relação com 0 cuidado de enfermagem, que precisa ser documentado, compreendido e utilizado de forma terapêutica (Leininger ${ }^{5,7}$ ).

0 objetivo da Enfermagem Transcultural transcende a simples apreciação de culturas diferentes. Ela é fundamentada na crença de que a maioria das culturas pode determinar o tipo de cuidado desejado direcionado para os usuários dos serviços de saúde. Também busca compreender a perspectiva desses usuários, sua visão de mundo, seu conhecimento e suas práticas como base para decisões e ações assistenciais. Neste sentido, Leininger ${ }^{1: 10}$ estabelece pressuposto básico sobre a profissão:

A enfermagem é um fenômeno essencialmente cultural que envolve o contexto e o processo de ajuda a indivíduos de diferentes orientaç̃oes culturais ou de estilo de vida específico, dentro de determinada cultura. 0 cuidado, as manifestações, os processos, os valores e as crenças de Enfermagem Transcultural precisam ser explicados de maneira sistemática e científica com bases humanísticas.

0 cuidado de enfermagem terapêutico e eficaz é culturalmente determinado e embasado nas necessidades do ser humano, podendo ser culturalmente validado. As culturas têm seu modo peculiar de comportamento relativo ao cuidado, que geralmente é conhecido pelos integrantes da própria cultura, mas freqüentemente desconhecido por enfermeiras com bagagem cultural diferente.

A busca de um equilibrio, que possibilite a harmonia de cada pessoa com seu próprio eu, é o caminho a trilhar na compreensão do corpo como totalidade que se encontra submetida à multiplicidade de aspectos, como a religião, lazer e a educação (Alves ${ }^{9}$ ). Nessa direção, fica clara a compreensão de que existem várias formas de cuidar, que nem sempre são explicáveis na sua dimensão biológica. Assim, as relações culturais estão entre as outras formas de conceber este cuidar.

A essência da Enfermagem é o cuidado e a essência da Enfermagem Transcultural é o cuidado para indivíduos com diversas heranças culturais relacionadas aos processos, às funções e às atividades, que variam de acordo com a estrutura social, o sistema cultural e a história cultural.
A Teoria da Diversidade e Universalidade do Cuidado Cultural apresenta vários conceitos: cultura, valor cultural, diversidade cultural de cuidado, universalidade cultural de cuidado, cuidado cultural, visão de mundo, estrutura social, contexto ambiental, sistema popular de saúde, saúde, sistema profissional de saúde, cuidar/cuidado, preservação cultural de cuidado, acomodação cultural do cuidado e repadronização cultural do cuidado(Leininger ${ }^{1}$ ).

Além desses conceitos, as culturas são os valores, crenças, ritos, normas e práticas de vida de um determinado grupo, aprendidos, partilhados e transmitidos que orientam o pensamento, as decisões e as ações, de maneiras padronizadas (Leininger ${ }^{5: 156}$ ). Um pressuposto relacionado à cultura é o de que os seres humanos são seres culturais capazes de sobreviver ao passar do tempo, através de sua capacidade de prestar cuidado aos outros, de todas as idades, em vários ambientes e de muitas maneiras. Os valores culturais derivam da cultura e identificam maneiras desejáveis de ação e conhecimento. Esses valores culturais são, comumente, mantidos pela cultura, por longos períodos de tempo, e servem para orientar a tomada de decisões dos membros da cultura (Leininger ${ }^{1}$ ).

Além das definições, Leininger:14 apresenta pressupostos que dão apoio à sua previsão de que culturas diferentes percebem e praticam cuidado de diferentes maneiras, ainda que alguns elementos comuns existam, em relação ao cuidado, em todas as culturas do mundo. Ela faz também referências aos elementos comuns como universalidade e às diferenças como diversidade. Assim, a diversidade cultural do cuidado indica a variação de significados, padrões, valores ou símbolos de cuidado que são culturalmente originários dos homens para seu bem-estar ou para aperfeiçoar uma condição ou modo de vida dos homens, ou para enfrentar a morte (Leininger:1:9). Por outro lado, o Cuidado Cultural é definido como:

Os valores, crenças e expressões padronizadas, cognitivamente conhecidos, que auxiliam, facilitam, sustentam ou capacitam outro indivíduo ou grupo a manter seu bem-estar, saúde, melhorar sua condição humana e seu modo de vida ou lidar com a doença, a deficiência ou a morte(Leiningerl: $\left.{ }^{13}\right)$.

0 pressuposto relacionado é que o cuidado cultural é o meio holístico e mais amplo para conhecer, explicar, interpretar e prever o fenômeno do atendimento de enfermagem visando orientar as práticas de cuidados de enfermagem (Leininger: 44). Verifica-se na prática da Enfermagem, a importância do conhecimento das enfermeiras em reconhecer as diferenças de padrões de comportamento de pessoas com bagagem cultural diversificada e a 
necessidade de contemplar individualmente 0 aspecto cultural no cuidado.

No que se refere à visão de mundo, o cuidado cultural é a maneira pela qual as pessoas olham o mundo ou o universo, e formam um "quadro ou atitude de valor" acerca do mundo e de suas vidas.

Contexto ambiental é a totalidade de um acontecimento, situação ou experiência particular que confere sentido às expressões humanas, incluindo interações sociais, dimensões físicas, ecológicas, emocionais e culturais (Leininger: ${ }^{11}$ ).

0 conhecimento, os significados e as práticas derivadas das visões de mundo, dos fatores de estrutura social, dos valores culturais, do contexto ambiental e dos usos da linguagem são essenciais para guiar a Enfermagem e as ações de seus profissionais no fornecimento de cuidado cultural coerente com a cultura da população.

No que se refere à saúde, ela se caracteriza por ser um estado de bem-estar que está culturalmente definido, valorizado e praticado e que reflete a capacidade que os indivíduos (ou grupos) possuem para realizar suas atividades diárias, de maneiras culturalmente satisfatórias (Leininger ${ }^{1}$ ). Continuando com esse pensamento, a autora faz uma distinção entre dois sistemas de saúde existentes na sociedade, sendo que um se refere aos sistemas de cuidados populares de saúde ou sistemas "folk", entendidos como:

Conhecimentos e habilidades tradicionais, populares (com base doméstica), culturalmente aprendidos e transmitidos, usados para proporcionar atos assistenciais, apoiadores, capacitadores ou facilitadores por ou para outro indivíduo, grupo ou instituição com necessidades evidentes ou antecipadas de melhorar um modo de vida humano, uma condição de saúde (ou bem-estar) ou para lidar com situações de deficiência ou morte (Leininger ${ }^{5: 158}$ ).

0 outro sistema de saúde é definido como sistema de cuidado ou de cura profissionais oferecidos por pessoal de saúde diversificado. Esse sistema trata da interpretação do vivido naquela cultura, na perspectiva de pessoas que não pertencem a ela e descrevem o cuidado do ponto de vista profissional; ou seja, um cuidado profissional formalmente ensinado, aprendido e transmitido, bem como o conhecimento de saúde, doença, bem-estar e as habilidades práticas que prevalecem em instituições educacionais especiais.

Além desses fatores relacionados com o cuidado, algumas definições devem ser destacadas, tais como: (1) Preservação Cultural do Cuidado, que implica em ações e decisões profissionais assistenciais, apoiadoras, facilitadoras ou capacitadoras, para auxiliar as pessoas de uma determinada cultura a reter e/ou conservar valores importantes de cuidados, de maneira que possam manter seu bem-estar, recuperar-se da enfermidade ou enfrentar as deficiências e/ou a morte; (2) Acomodação Cultural do Cuidado, que engloba ações e decisões profissionais assistenciais criativas, apoiadoras, facilitadoras ou capacitadoras, que auxiliam as pessoas de uma determinada cultura a se ajustar ou negociar com outras um resultado de saúde benéfico ou satisfatório com os responsáveis pelos cuidados profissionais; (3) Reestruturação do Cuidado Cultural, que junta as ações e decisões profissionais assistenciais apoiadoras, facilitadoras ou capacitadoras, que auxiliam o cliente a reorganizar, permutar ou modificar sua maneira de vida para um novo padrão de atendimento à saúde. A esse respeito, a reestruturação requer o uso criativo de um enorme conhecimento da base cultural do cliente e deve ser efetuada com base em seu modo de vida, enquanto são usados o conhecimento popular e o profissional (Leininger ${ }^{1}$ ).

No contexto do estudo dos rituais de saúde, Leininger ${ }^{1}$ destaca que o foco sobre os rituais-de-cuidado-de-vida e os rituais de passagem não têm sido muito considerados pelos estudiosos. No entanto, essa abordagem é extremamente importante para se realizar avaliações "culturológicas", uma vez que, em quase todos os grupos culturais do mundo, existem cuidados de vida apoiados em rituais.

Segundo Monticelli ${ }^{10}$, uma das abordagens mais antigas sobre ritos foi a de Gennep ${ }^{11}$, em seu estudo clássico intitulado "Os ritos de passagem", publicado em 1908, na França. Essa abordagem ainda continua sendo utilizada por pesquisadores, não só da antropologia como também de outras áreas, tanto no Brasil quanto no exterior. No Brasil, o trabalho de Gennep foi publicado em 1978, observando-se, a partir daí, sua influência em estudos com a temática sobre os rituais brasileiros (Monticelli ${ }^{10}$ ).

Monticelli ${ }^{10}$ prossegue destacando a contribuição dos estudos de Turner ${ }^{12}$ para a abordagem dos rituais em trabalhos relacionados às ciências sociais, incluindo-se a área da saúde. Este autor vê no processo ritual a possibilidade de atualização da anti-estrutura, estados potenciais e liminares da sociedade, que constrói, assim, sua transformação, expondo as variantes possíveis de seu destino no que ele chama de drama-social (Turner ${ }^{12: 67}$ ).

A concepção de Turner ${ }^{12}$ implica também numa visão do ritual como um processo dinâmico de mudança de uma para outra estrutura e de um estado social para outro. Nessa direção, Rodrigues ${ }^{13}$, ao se referir ao processo de nascimento, comenta que ele é embebido culturalmente e que os procedimentos com o recém-nascido têm a função tanto de promover a sua transição do estado de natureza 
para o estado de cultura, quanto de proporcionar ritos de cuidados que possibilitem o início de seu processo de viver. Igualmente, pode-se analisar tanto a transição da saúde para a doença, como as transições ou fases do ciclo de vida pelas quais passa o ser humano, durante o seu processo de viver.

A esse respeito, Leininger ${ }^{1}$ esclarece que as ações desenvolvidas pela Enfermagem são baseadas num corpo de conhecimentos que englobam a cultura pessoal e profissional. Assim, a visão do processo saúde-doença dos profissionais nem sempre reflete a visão de saúde-doença dos seres humanos com os quais interagem. Por esse motivo, os ritos de cuidado populares e profissionais podem ser conflitantes durante o processo do nascimento. Cabe à enfermeira refletir sobre essas diferenças juntamente com outras mulheres, para definir o estabelecimento de ritos de cuidado culturalmente congruentes.

Das várias literaturas mencionadas, pode-se depreender que a abordagem ritual no campo da saúde, embora importante no sentido de trazer contribuições à área, não tem sido objeto sistemático de estudo. No campo da Enfermagem, alguns trabalhos vêm sendo realizados. No entanto, a maior parte deles destaca o trabalho interno dos enfermeiros e a estrutura do cuidado em ambientes hospitalares que, na maioria das vezes, têm uma conotação exploratória e um caráter técnico e funcionalista.

Especificamente no Brasil, quase inexistem estudos desta natureza, o que aponta para uma lacuna de conhecimentos no que diz respeito à abordagem dos rituais de cuidado que são desenvolvidos pelos próprios indivíduos, famílias e grupos em seus contextos culturais. Se, por um lado, os estudiosos de enfermagem têm colocado ênfase na necessidade desses estudos, por outro as pesquisas dessa natureza não têm sido merecedoras da mesma atenção.

Nesse sentido, concordamos com Monticelli ${ }^{10}$ quando esta autora ressalta que os rituais de saúde podem ser de grande importância para a prática da enfermagem, pois permitem analisar o comportamento das pessoas em relação ao corpo, à saúde e doença e à vida e morte, em qualquer grupo ou comunidade. Permitem, também, analisar a própria estrutura, as normas e rotinas estabelecidas em instituições de saúde e a dinâmica interna da Enfermagem e dos profissionais de saúde.

Pelo exposto, a abordagem cultural não deixa dúvidas de que os ritos estão indissociavelmente ligados ao processo saúde-doença envolvendo questões de ordem biológica, afetiva, social e comunicativa. Assim, os ritos são indispensáveis para que os profissionais de saúde, e particularmente as enfermeiras, atuem de forma a compreender a visão de mundo dos seres humanos com quem estão envolvidos na assistência à saúde.

\section{A TEORIA TRANSCULTURAL E SUA APLICAÇÃO EM ALGUMAS PESQUISAS DE ENFERMAGEM}

Patrício $^{14}$ destacou, na prática do cuidar/cuidado à família da adolescente grávida solteira e seu recémnascido, o referencial teórico da Enfermagem Transcultural para famílias dessas adolescentes oriundas de áreas da periferia urbana, bem como do estrato social médio. Esta autora afirma que, nas situações em que não é possível preservar o cuidado, poderá ser realizada uma acomodação, isto é, a união dos modos de cuidar do enfermeiro com aqueles do cliente.

Da mesma forma, o relato de experiência de Castanhel e Boehs ${ }^{15}$, com base na aplicação da Teoria do Cuidado Cultural, descreve a assistência para uma família imigrante, cujos membros estavam desempregados, viviam de esmolas e residiam em área de invasão na periferia urbana.

Outra pesquisa sobre o cliente com infarto agudo do miocárdio, com base na Teoria da Diversidade e Universalidade do Cuidado Cultural, descreve a experiência da elaboração, implementação e análise de um referencial para guiar o cuidado de enfermagem voltado para essa clientela, em uma unidade de terapia intensiva $\left(\right.$ Azevedo $\left.{ }^{16}\right)$.

Assim, pode-se verificar que no período de 1975 a 1983, houve um maior incremento do interesse pela Enfermagem Transcultural ocorrendo uma valorização por parte das enfermeiras. Esse período foi marcado por um aumento substancial de publicações, cujos autores apresentavam preparo antropológico e estavam familiarizados com métodos de pesquisa de campo.

Na prática, o processo de enfermagem baseado nesta Teoria está composto por várias etapas, nas quais a enfermeira aproxima-se do cliente identificando os cuidados culturais e desenvolvendo as ações de cuidados, ora os preservando, ora os acomodando ou ora os repadronizando. Leininger'diz que o processo avalia, com os clientes, os cuidados e/ou as ações desenvolvidas com vistas à prestação de um cuidado cultural. Esta abordagem teórica contribuiu para ampliar a visão do campo de atuação da Enfermagem, ao vislumbrar a possibilidade de aproximar a cultura profissional/institucional à cultura do cliente.

\section{COMENTÁRIOS FINAIS}

0 grande desafio das enfermeiras e dos enfermeiros que se aventuram pelos caminhos da antropologia para cuidar do ser humano de uma forma mais abrangente é, dentro da cultura da Enfermagem, eliminar a armadura do modelo profissional, por alguns momentos, e ver o cliente no seu mundo, entender suas ações e, ao mesmo tempo, não deixar de ser 
enfermeiro ou enfermeira. Apesar das inquietações que podem levar os profissionais a questionar como é ser um e outro ao mesmo tempo, é necessário perceber o conhecimento e transpô-lo, bem como conhecer também a cultura do sujeito a que se assiste.

A Teoria da Diversidade e Universalidade do Cuidado Cultural é uma proposta que pode vencer este desafio. Mas, por ser uma teoria abrangente, ela tem conceitos abstratos, havendo um amplo campo para dar mais respostas ao desafio de como utilizar esta visão antropológica na Enfermagem e o significado cultural do cuidado.

No Brasil, esta Teoria tem aproximado mais as enfermeiras da antropologia. Sem dúvida, utilizar uma teoria com base na antropologia e na enfermagem é uma contribuição para aproximar o mundo do cliente do mundo do profissional da saúde e fomentar o conhecimento na Enfermagem. Para que isto ocorra, devemos estar aten-

\section{Referências}

1. Leininger M, Farland MR. Transcultural nursing: concepts, theories,

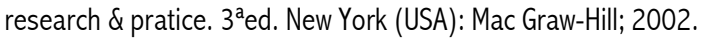

2. Minayo MCS. Abordagem antropológica para avaliação de políticas sociais. Rev Saúde Pública 1991; 25(3): 233-8.

3. Langdon EJ. 0 processo de ser saudável sob o ponto de vista antropológico. Florianópolis (SC): Centro de Ciências da Saúde/ UFSC; 1993. Notas de seminário.

4. Queiroz MV. Só a mãe conhece o filho: um estudo na etnoenfermagem. [dissertação de mestrado]. Fortaleza (CE); Escola de Enfermagem / UFCE; 1998. 97 p.

5. Leininger M. Leininger's teory of nursing: cultural care diversity and universality. Nurs Sci Q 1998; 1: 152-60.

6. Leininger M. Ethnomethods: the philosophic and epistemic bases to explicate transcultural nursing. J Transcult Nurs 1990; 1(2): 40-52.

7. Leininger M. Culture care diversity and universality: a theory of nursing. New York (USA): National League for Nursing; 1991.

8. Herberg P. Theoretical foundations of transcultural nursing. In: Andrews MM, Boyle JS. Transcultural concepts in nursing care. $2^{\mathrm{a}}$ ed. Philadephia (USA): Lippincott; 1995. p. 3-48 . cap.1 tos para aplicar uma teoria de maneira não repetitiva e com avaliações críticas, para não incorrer no risco de investir em vão. Esta Teoria, bem como outras abordagens com base na antropologia, tem limitações, ambigüidades e termos que precisam ser esclarecidos dentro do contexto do campo de pesquisa, no qual são aplicados.

Convém finalmente destacar as duas contribuições que o presente trabalho pretende oferecer àqueles que atuam na assistência de enfermagem. Uma delas diz respeito às experiências já existentes na aplicação da Teoria aqui abordada, seja na prática ou na pesquisa e à luz da literatura crítica que vem despontando. Entendemos que as enfermeiras, ao utilizarem a Enfermagem Transcultural ou outra ferramenta baseada na antropologia, possam avançar no sentido de pensar esta abordagem dentro do contexto real, local e nacional. A outra contribuição, na verdade, é a sincera pretensão de que este trabalho, ainda que limitado, dê origem a outras reflexões sobre a temática.

9. Alves AR et al. Saúde e doença: uma abordagem sócio cultural. In: Silva YF, Franco MC. Saúde e doença: uma abordagem cultural da enfermagem. Florianópolis (SC): Papa Livro; 1996.

10. Monticelli M. Nascimento como rito de passagem: abordagem cultural para o cuidado de enfermagem às mulheres e aos recémnascidos. [dissertação de mestrado]. Escola de Enfermagem/UFSC; 1994.

11. Gennep AV. Os ritos de passagem. Petrópolis (RJ): Vozes; 1978.

12. Turner V. Dramas, fields and metaphors. Ithaca (USA): Cornell University Press; 1974.

13. Rodrigues NE et al. Saúde em risco. Revista Debate 1989; 22: 37-38.

14. Patrício ZM. A prática do cuidar/cuidado à família da adolescente grávida solteira e ser recém-nascido através de um marco conceitual de enfermagem de enfoque sociocultural. [dissertação de mestrado]. Faculdade de Enfermagem/ UFSC; 1990.

15. Castanhel M, Boehs AE. Cuidando de uma família na comunidade: uma experiência utilizando um referencial teórico. Texto \& Contexto Enferm 1993 jul/dez ;2(2): 87-98.

16. Azevedo RCS. Possibilidades de uma abordagem cultural no cuidado ao cliente com enfarto agudo do miocárdio. [dissertação de mestrado]. Mato Grosso (MT): Universidade Federal de Mato Grosso; 1998. 136 p. 


\section{Sobre as Autoras}

\section{Maria Aparecida Vasconcelos Moura}

Professora, Doutora e Pesquisadora do Núcleo de Pesquisa em Enfermagem em Saúde da Mulher, do Departamento de Enfermagem Materno-Infantil e Coordenadora Adjunta do Curso de Mestrado da Escola de Enfermagem Anna Nery / Universidade Federal do Rio de Janeiro.

E-mailmaparecidavas@yahoo.com.br.

\section{Rosilda Alves da Silva Isla Chamilco}

Professora, Doutora e Coordenadora do Curso de Graduação em Enfermagem da Universidade Federal do Amapá.

E-mail chamilco@yahoo.com.br.

\section{Leila Rangel da Silva}

Professora, Doutora e Coordenadora do Núcleo de Pesquisa, Experimentação e Estudos na Área da Saúde da Mulher e da Criança, do Departamento de Enfermagem Materno-Infantil da Escola de Enfermagem Alfredo Pinto da Universidade Federal do Estado do Rio de Janeiro.

E-mail: Irangel@rio.com.br 